\title{
Structure of Halo and Quasi-Halo Helium-Helium-Alkali Trimers
}

\author{
Petar Stipanović · Leandra Vranješ Markić · Jordi Boronat
}

Received: date / Accepted: date

\begin{abstract}
We report a diffusion Monte Carlo study of $\mathrm{A}^{4} \mathrm{He}_{2}$ and $\mathrm{A}^{4} \mathrm{He}^{3} \mathrm{He}$ trimers' structural properties, were $\mathrm{A}$ is one of the alkali atoms ${ }^{6,7} \mathrm{Li},{ }^{23} \mathrm{Na},{ }^{39} \mathrm{~K},{ }^{85} \mathrm{Rb}$ or ${ }^{133} \mathrm{Cs}$. Some of them are in a pure halo state, characterized by large spatial extent and universality, while some are close to the halo limit. The theoretical analysis of these trimers enables insight on how structural properties of weakly bound systems change when approaching the halo edge. For that purpose, two-variable distributions of inter-particle separations and angles were calculated. Extreme spatial extensions of some trimers with ${ }^{3} \mathrm{He}$ confirm their halo nature. Although all the considered systems are floppy, trimers with all bound dimer subsystems are less spread and have significantly lower percentage of quasi-linear configurations than those which have at least one unbound dimer subsystem.
\end{abstract}

Keywords halo states $\cdot$ three-body $\cdot$ helium-alkali clusters structure $\cdot$ quantum Monte Carlo

PACS 02.70.Ss $\cdot 36.40 .-\mathrm{c} \cdot 67.90 .+\mathrm{z}$

\section{Introduction}

Weakly bound and spatially extended few-body systems have been attracting considerable interest since they share universal features. This key characteristic enables comparison

Petar Stipanović

University of Split, Faculty of Science, R. Boškovića 33, HR-21000 Split, Croatia

Tel.: +38521619234

E-mail: pero@pmfst.hr

Leandra Vranješ Markić

University of Split, Faculty of Science, R. Boškovića 33, HR-21000 Split, Croatia

Jordi Boronat

Departament de Física, Campus Nord B4-B5, Universitat Politècnica de Catalunya, E-08034 Barcelona, Spain of systems from different fields of physics, from ultracold gases to nuclear physics. Specifically, in quantum three-body physics the most famous property is the Efimov effect [1]. An infinite series of three-body geometrically spaced and stable excited states was predicted [1], and latter experimentally confirmed [2], to appear when a third particle is added to a pair of bosons that are on the edge of binding. Universality is also related to the two- and three-body systems, which mostly occupy classically forbidden regions of space, the so called halo states [3-5]. Such systems are not sensitive to details of the short-range interactions. Thus, their properties can be predicted with any potential that has the same integral properties, i.e., the scattering length and the effective range. The scale invariance noticed in nuclei [3] was confirmed also for small atomic clusters $[3,5]$. Those works proposed a universal relation between scaled size and energy for two- and three-body systems as well as separation of lines corresponding to different types of trimers. Namely, trimers can be classified as Borromean [3], tango [6], samba [7] and all-bound type if they have zero, one, two and all three two-body subsystems bound, respectively. Universal relations between scaled size and energy of weakly bound threebody systems were calculated in three [8] and two [9] spatial dimensions.

Due to their extremely weak interaction, both helium isotopes, ${ }^{4} \mathrm{He}$ and ${ }^{3} \mathrm{He}$ played a significant role in the study of molecular halos $[3,5]$. Furthermore, it was experimentally confirmed that the excited state of ${ }^{4} \mathrm{He}_{3}$ is an Efimov state, while the ground state of ${ }^{4} \mathrm{He}_{2}{ }^{3} \mathrm{He}$ was suggested to be a quantum halo state $[10,11]$. Recently, helium trimer properties have been thoroughly investigated not only theoretically [12-14], but also experimentally [12,13]. Since Healkali potentials are even shallower, one expects that swapping a He isotope by an alkali could produce a halo state or a diffuse system close to the halo border (quasi-halo). Very weak binding of mixtures $\mathrm{HeHeA}$, where $\mathrm{A}$ is one of 
the alkali atoms, was predicted by different methods [1520,22,21]. In addition, the first excited states of ${ }^{4} \mathrm{He}_{2}{ }^{6} \mathrm{Li}$ and ${ }^{4} \mathrm{He}_{2}{ }^{7} \mathrm{Li}$ were predicted to be Efimov states. [15-19,21]. Also recently, weak binding of ${ }^{4} \mathrm{He}_{2} \mathrm{~A}$ has been confirmed [23-25]. Swapping a ${ }^{4} \mathrm{He}$ by ${ }^{3} \mathrm{He}$ generates even more diffuse clusters, showing a pure halo characteristics [5,25].

However, structural properties of helium-alkali clusters have not been thoroughly investigated so far. In particular, two-dimensional angular and radial distribution functions, which would give better insight into the different triangular configurations, are lacking. Since helium-alkali trimers offer a huge set of weakly bound systems under natural conditions, we analyzed their structure in more detail to test how mass and binding strength affect their diffuseness in different directions. In particular, we notice the difference in trimer configuration belonging to different types, all-bound and tango/samba trimers.

Since very weakly bound system are computationally quite demanding, we chose the diffusion Monte Carlo method (DMC) [26] and pure estimators [27] to extract exact groundstate properties. The method is discussed in Sec. 2. Sec. 3 reports the distribution functions. Finally, Sec. 4 comprises a summary of the work and an account of the main conclusions.

\section{Method}

Clusters $\mathrm{A}^{4} \mathrm{He}_{2}$ and $\mathrm{A}^{4} \mathrm{He}^{3} \mathrm{He}$ were studied at zero temperature using the DMC method. The DMC method solves stochastically the Schrödinger equation written in imaginary time $\tau=i t / \hbar$,

$$
-\frac{\partial \Psi(\mathbf{R}, \tau)}{\partial \tau}=\left(H-E_{\mathrm{r}}\right) \Psi(\mathbf{R}, \tau)
$$

applying reasonable approximations for the Green's function when imaginary time step $\Delta \tau \rightarrow 0$. In Eq. (1), $E_{\mathrm{r}}$ is a reference energy and the walker $\mathbf{R} \equiv\left(\mathbf{r}_{1}, \mathbf{r}_{2}, \mathbf{r}_{3}\right)$ collectively denotes the positions of the trimer's constituents.

The Hamiltonian $H$ for the trimer is

$$
H=-\sum_{i=1}^{3} \frac{\hbar^{2}}{2 m_{i}} \nabla_{\mathbf{i}}^{\mathbf{2}}+\sum_{\substack{\mathbf{i}, \mathbf{j}=\mathbf{1} \\ \mathbf{i}<\mathbf{j}}}^{\mathbf{3}} \mathbf{V}\left(\mathbf{r}_{\mathbf{i j}}\right),
$$

where $V$ denotes the interatomic potentials between the three pairs of the trimer. Explicit three-body potentials are not included because their contribution has proved to be negligible in the case of pure He trimers [13,28]. For He-He interactions we used the semi-empirical HFDB potential [29] as in Refs. [5,13] and for He-A the KTTY [30] model as in Ref. [5]. The best available constants from the NIST database were taken,

$$
0.5 \hbar^{2} m^{-1} /\left(\mathrm{mK} \AA^{2}\right)
$$

for ${ }^{3} \mathrm{He},{ }^{4} \mathrm{He},{ }^{6} \mathrm{Li},{ }^{7} \mathrm{Li},{ }^{23} \mathrm{Na},{ }^{39} \mathrm{~K},{ }^{85} \mathrm{Rb}$, and ${ }^{133} \mathrm{Cs}$, respectively equal to $8041.811058,6059.640786,4032.226566$, $3457.001455,1055.005713,622.4853844,285.6415818$, and 182.4931752 .

In order to solve Eq.(1) we applied the same procedure as in Refs. [13,25] In particular, the details of the calculations are given in the work that reports the stability and onedimensional distribution functions of ${ }^{4} \mathrm{He}_{2} \mathrm{~A},{ }^{4} \mathrm{He}^{3} \mathrm{HeA}$ and ${ }^{3} \mathrm{He}_{2} \mathrm{~A}$, where $\mathrm{A}$ is one of the alkali atoms $\mathrm{Li}, \mathrm{Na}, \mathrm{K}, \mathrm{Rb}$ or Cs. [25]

For the calculation of the atom pair-pair distributions $P\left(r_{i j}, r_{k l}\right)$ and angular-angular distributions $P\left(\theta_{1}, \theta_{3}\right)$ it was necessary to use pure estimators [27], because the corresponding operators do not commute with the Hamiltonian $H$. In order to ensure exact ground-state properties, it is crucial in this algorithm to verify that the chosen block size is large enough. We used $10^{5}$ steps per block for all studied structural properties, although for some trimers they converged even for 3 times smaller block sizes. For the calculations of the distribution functions we have chosen a time step $\Delta \tau=10^{-3} \mathrm{~K}^{-1}$ that we checked to be small enough to reduce any finite time-step bias.

\section{Results}

Motivated by the recent experimentally measured $[10,11$, 31] distributions of helium trimers ${ }^{4} \mathrm{He}_{3}$ and ${ }^{4} \mathrm{He}_{2}{ }^{3} \mathrm{He}$, we determined the structural properties of the ground state for all $\mathrm{A}^{4} \mathrm{He}_{2}$ and $\mathrm{A}^{4} \mathrm{He}^{3} \mathrm{He}$ trimers. The structure is visualized by two-variable distribution functions, inter-particle separations $P\left(\mathrm{He}-\mathrm{He},{ }^{4} \mathrm{He}-\mathrm{A}\right)$ and angles $P\left(\theta_{1}, \theta_{3}\right)$ where $\theta_{1}$ and $\theta_{3}$ denote respectively $\alpha$ and $\gamma$ angle of the triangle ABC formed by atoms.

Results for the distribution functions are shown in figures 1, and 2. Top rows present $\mathrm{A}^{4} \mathrm{He}^{3} \mathrm{He}$ trimers, while $\mathrm{A}^{4} \mathrm{He}_{2}$ are in a bottom rows. In Fig. 1, the separation between He-He atoms is in the $x$-axis, while the ${ }^{4} \mathrm{He}-\mathrm{A}$ distances are in the $y$-axis. The repulsive barrier of the He-A potential is wider than the He-He one, producing a wider zero probability area extending next to the $x$-axis than next to the $y$-axis. Color-boxes for top and bottom row are different for clarity reasons because trimers $\mathrm{A}^{4} \mathrm{He}^{3} \mathrm{He}$ are much more diffuse than $\mathrm{A}^{4} \mathrm{He}_{2}$, which are not in a halo state [25]. Our previous study has shown that universal scaling of energy and size [5] extends also somewhat below the halo domain. Similar distributions for $\mathrm{A}^{4} \mathrm{He}_{2}$ suggest that they should be concentrated very close in the scaling plot from Ref. [5]. Higher peaks mean higher localization which is in agreement with the predicted binding energies [14,25]. Swapping the heavier He isotope by the lighter, the effect becomes more pronounced. All $\mathrm{A}^{4} \mathrm{He}^{3} \mathrm{He}$ trimers are tango states, except the one with $\mathrm{Na}$, which is a samba state because the ${ }^{3} \mathrm{He}-\mathrm{Na}$ dimer is bound with the energy of -1.242 


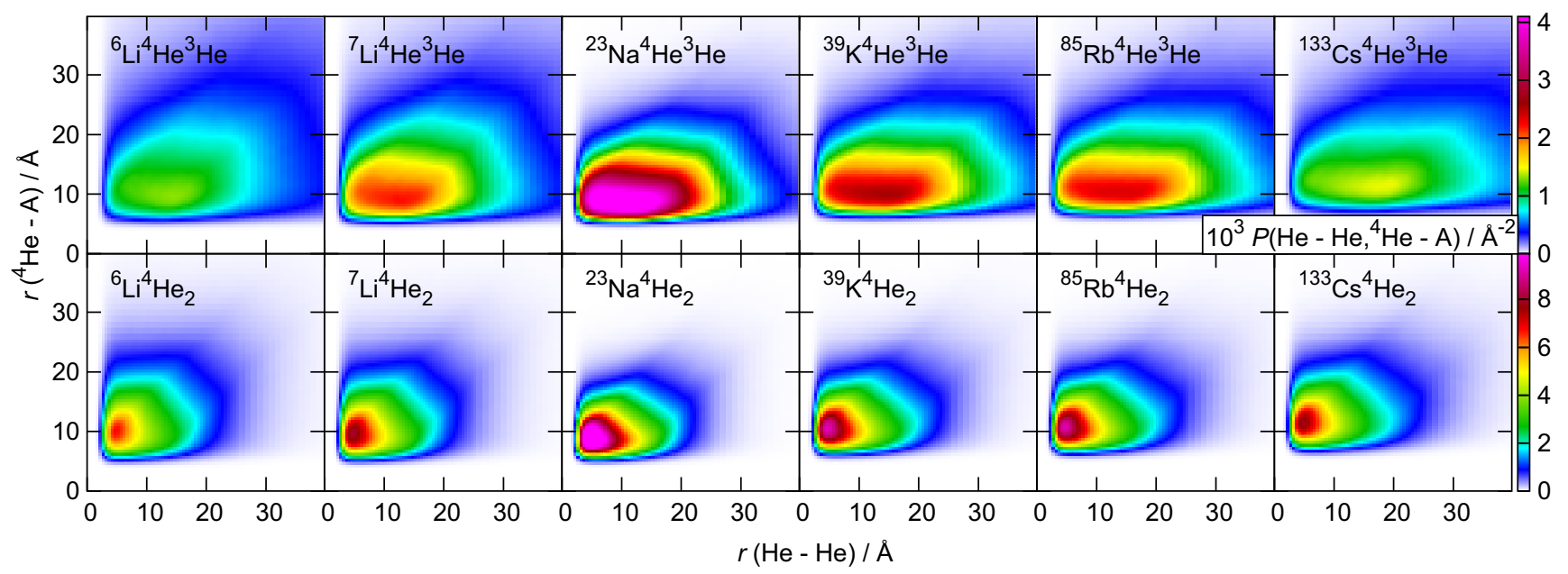

Fig. 1 Distributions of $\mathrm{He}-\mathrm{He}$ and ${ }^{4} \mathrm{He}-\mathrm{A}$ separations in: $\mathrm{A}^{4} \mathrm{He}^{3} \mathrm{He}$ (top) and $\mathrm{A}^{4} \mathrm{He}_{2}$ (bottom), where $\mathrm{A}$ is an alkali metal; $\int P \mathrm{~d}^{2} r=1$

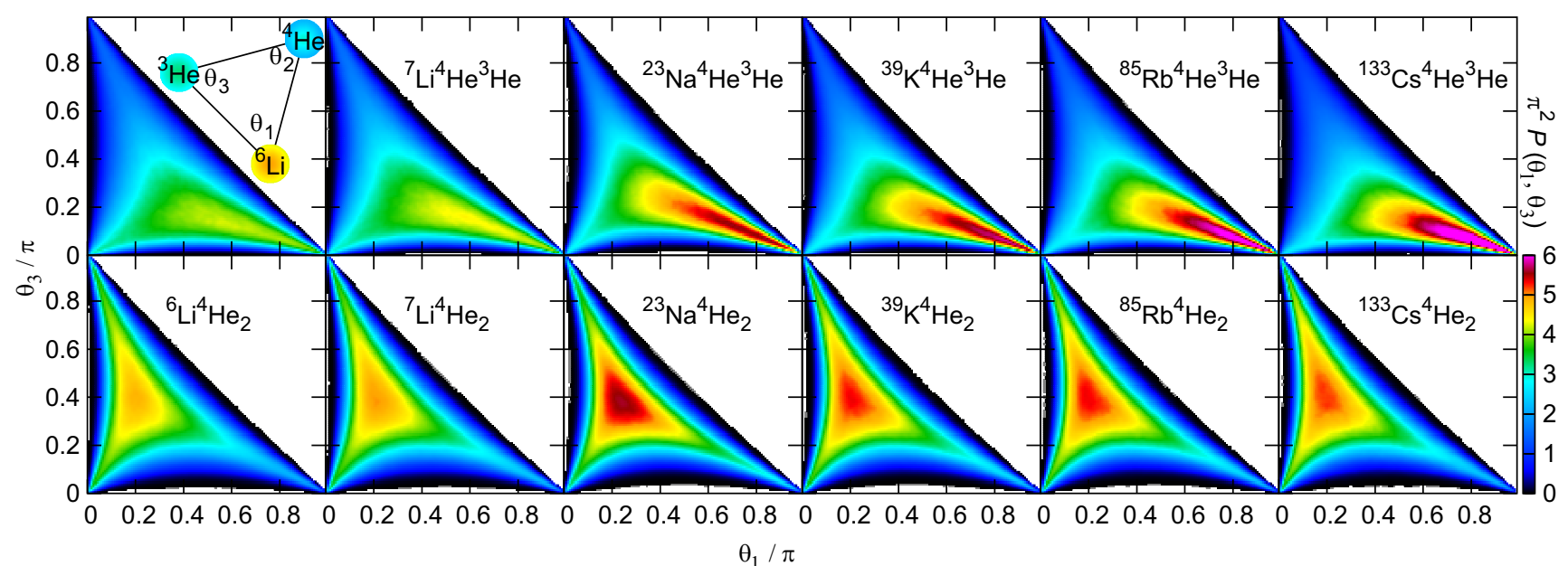

Fig. 2 Distributions of angles $\theta_{1}=\measuredangle\left({ }^{3,4} \mathrm{He}-\mathrm{A}-{ }^{4} \mathrm{He}\right)$ and $\theta_{3}=\measuredangle\left({ }^{4} \mathrm{He}-{ }^{3,4} \mathrm{He}-\mathrm{A}\right)$ in helium-alkali trimers: $\mathrm{A}^{4} \mathrm{He}{ }^{3} \mathrm{He}$ (top) and $\mathrm{A}^{4} \mathrm{He} 2$ (bottom), normalized to $\int P\left(\theta_{1}, \theta_{3}\right) \mathrm{d} \theta_{1} \mathrm{~d} \theta_{3}=1$

$\mathrm{mK}$. Larger differences in distributions are in agreement with trimers' spread along the universal tango line [5]. E. g. scaled sizes of 3.3 and 1.7 were obtained [5] for ${ }^{3} \mathrm{He}^{4} \mathrm{He}^{7} \mathrm{Li}$ and ${ }^{3} \mathrm{He}^{4} \mathrm{He}^{39} \mathrm{~K}$, respectively, what classifies them as halo and quasi-halo close to the halo limit 2 . All clusters are squeezed in the direction of the axis belonging to the heavier pair, $y$ axis. The effect is the more pronounced the stronger binding of the He-A pair is with respect to the the He-He binding (-1.69 mK). Namely, ${ }^{4} \mathrm{He}-\mathrm{A}$ binding energies in $\mathrm{mK}$ are $-1.515,-5.622,-28.98,-11.2,-10.27,-4.954$ for ${ }^{6} \mathrm{Li},{ }^{7} \mathrm{Li}$, ${ }^{23} \mathrm{Na},{ }^{39} \mathrm{~K},{ }^{85} \mathrm{Rb}$ and ${ }^{133} \mathrm{Cs}$, respectively. Manifolded system's configuration arrangements are noticeable in all trimers, i.e., for a particular $r_{i j}$ value a wide range of distances $r_{k l}$ has high probability of occurrence.

Angle-angle distribution functions shown in Fig. 2 provide additional insight into the possible types of triangular configurations. Angle $\theta_{1}=\measuredangle\left({ }^{3,4} \mathrm{He}-\mathrm{A}-{ }^{4} \mathrm{He}\right)$ is shown on $x$-axis, while $y$-axis stands for $\theta_{3}=\measuredangle\left({ }^{4} \mathrm{He}-{ }^{3,4} \mathrm{He}-\mathrm{A}\right)$. Angles are marked for ${ }^{6} \mathrm{Li}^{4} \mathrm{He}^{3} \mathrm{He}$ in upper right corner of the first subfigure in the top row. All of the trimers are spread among different possible triangular shapes. As the mass of the alkali atom is increased a more defined maximum starts to form. The overall symmetry is significantly different in the cases of $\mathrm{A}^{4} \mathrm{He}^{3} \mathrm{He}$ trimers, presented in the upper row and $\mathrm{A}^{4} \mathrm{He}_{2}$ ones in the bottom row. In $\mathrm{A}^{4} \mathrm{He}^{3} \mathrm{He}$ trimers, which have at least one unbound subsystem, when the A mass in increased, a maximum forms with the small value of the angle at the ${ }^{3} \mathrm{He}$ atom and the large angle at the A atom, suggesting a significant proportion of nearly linear configurations. On the other hand, in the case of $\mathrm{A}^{4} \mathrm{He}_{2}$ such configurations have very low probability. The most probable triangular configuration are given in Table 1. Isosceles configurations are preferred by $\mathrm{A}^{4} \mathrm{He}_{2}$, with approximately two times smaller angle in the A corner than in the He one. In contrast to that, in the $\mathrm{A}^{4} \mathrm{He}^{3} \mathrm{He}$ trimer, the angle in the ${ }^{4} \mathrm{He}$ vertex is two times larger than in the ${ }^{3} \mathrm{He}$ one while the angle $\theta_{1}$ increases with the A mass from $97^{\circ}$ to $165^{\circ}$. 
Table 1 Most probable triangular configuration of atoms in trimers $\mathrm{A}-{ }^{4} \mathrm{H}-{ }^{4} \mathrm{He}$ and $\mathrm{A}-{ }^{4} \mathrm{He}-{ }^{3} \mathrm{He}$, given with the uncertainty of $5^{\circ}$

\begin{tabular}{ccc}
\hline & \multicolumn{2}{c}{$\theta_{1}-\theta_{2}-\theta_{3}\left[^{\circ}\right]$ in $\mathrm{A}-\mathrm{B}-\mathrm{C}$} \\
\cline { 2 - 3 } $\mathrm{A}$ & $\mathrm{A}-{ }^{4} \mathrm{H}-{ }^{4} \mathrm{He}$ & $\mathrm{A}-{ }^{4} \mathrm{He}-{ }^{3} \mathrm{He}$ \\
\hline${ }^{6} \mathrm{Li}$ & $36-71-73$ & $097-59-24$ \\
${ }^{7} \mathrm{Li}$ & $37-74-69$ & $103-48-29$ \\
${ }^{23} \mathrm{Na}$ & $43-70-67$ & $150-19-11$ \\
${ }^{39} \mathrm{~K}$ & $34-76-70$ & $159-14-07$ \\
${ }^{85} \mathrm{Rb}$ & $33-74-73$ & $159-14-07$ \\
${ }^{133} \mathrm{Cs}$ & $34-80-66$ & $165-11-04$ \\
\hline
\end{tabular}

\section{Conclusions}

Using accurate diffusion Monte Carlo simulations we obtained the ground-state structural properties for all trimers $\mathrm{A}^{4} \mathrm{He}_{2}$ and $\mathrm{A}^{4} \mathrm{He}^{3} \mathrm{He}$, where $\mathrm{A}$ is an alkali metal ${ }^{6,7} \mathrm{Li},{ }^{23} \mathrm{Na}$, ${ }^{39} \mathrm{~K},{ }^{85} \mathrm{Rb}$ or ${ }^{133} \mathrm{Cs}$. Structural properties were calculated using pure estimators in order to completely eliminate the bias of the trial wavefunction used for guiding the diffusion process within the DMC method.

Our results for the distribution of $\mathrm{He}-\mathrm{He}$ and ${ }^{4} \mathrm{He}-\mathrm{A}$ distances show a noticeable squeezing of the $\mathrm{HeHeA}$ cluster in the He-A direction. The effect is the more pronounced the stronger binding of the He-A pair is in comparison to the HeHe binding. Samba and tango type trimers are more spatially stretched than all-bound trimers. From angular-angular distributions it is noticeable that samba ${ }^{23} \mathrm{Na}^{4} \mathrm{He}^{3} \mathrm{He}$ and $\mathrm{A}^{4} \mathrm{He}^{3} \mathrm{He}$ tango-type trimers show with large probability triangular configurations close to the stretched triangle with $\mathrm{A}$ in the middle. The unbound $\mathrm{He}-\mathrm{He}$ pair enables their appearance. In contrast to that, all-bound trimers barely experience these configurations since all bound pairs prevent stretching. Increasing the binding energy, the distributions become more classical, although the high localizations of angles around a particular most probable configuration, as in $\mathrm{Ne}_{3}$ [14] is still not observed.

The one-variable distributions of inter-particle distances and angles in ${ }^{4} \mathrm{He}_{3}$ and ${ }^{4} \mathrm{He}_{2}{ }^{3} \mathrm{He}$ trimers have been recently measured. $[10,11]$ This opens up the possibility that manyvariable distributions could be also extracted. Since they give more information about the system, comparison of theoretical and experimental results would help to know if theoretical models need additional refinements. Particularly, there are significantly different He-A model potentials in the literature. We chose the KTTY model [30] since it was recently used in the construction of a universal potential model [32] and was mostly used in previous few-body calculations. Anyway, any experimental confirmation of its quality would be very welcome from the theoretical side.

Acknowledgements This work has been supported in part by the Croatian Science Foundation under the project number IP-2014-09-2452. J. B. acknowledge additional support by the MICINN-Spain, Grant No.
FIS2014-56257-C2-1-P. The computational resources of the Isabella cluster at Zagreb University Computing Center (Srce), the HYBRID cluster at the University of Split, Faculty of Science and Croatian National Grid Infrastructure (CRO NGI) were used.

\section{References}

1. V. Efimov, Phys. Lett. 33B, 563 (1970)

2. T. Kraemer, M. Mark, P. Waldburger, J. G. Danzl, C. Chin, B. Engeser, A. D. Lange, K. Pilch, A. Jaakkola, H.C. Nägerl, R. Grimm, Nature 440, 315 (2006)

3. A. S. Jensen, K. Riisager, D. V. Fedorov, E. Garrido, Rev. Mod. Phys. 76, 215 (2004)

4. K. Riisager, Phys. Scr. T152, 014001 (2013)

5. P. Stipanović, L. Vranješ Markić, I. Bešlić, J. Boronat, Phys. Rev. Lett. 113, 253401 (2014)

6. F. Robicheaux, Phys. Rev. A 60, 1706 (1999)

7. M. T. Yamashita, L. Tomio, T. Frederico, Nuclear Phys. A 735, 40 (2004)

8. M. T. Yamashita, R. S. Marques de Carvalho, Lauro Tomio, and T. Frederico, Phys. Rev. A 68, 012506 (2003)

9. J. H. Sandoval, F. F. Bellotti, A. S. Jensen, and M. T. Yamashita, Phys. Rev. A 94, 022514 (2016)

10. J. Voigtsberger, S. Zeller, J. Becht, N. Neumann, F. Sturm, H-K. Kim, M. Waitz, F. Trinter, M. Kunitski, A. Kalinin, J. Wu, W. Schöllkopf, D. Bressanini, A. Czasch, J. B. Williams, K. UllmannPfleger, L. Ph H. Schmidt, M. S. Schöffler, R. E. Grisenti, T. Jahnke, R. Dörner, Nature Communications 5, 5765 (2014)

11. M. Kunitski, S. Zeller, J. Voigtsberger, A. Kalinin, L. Ph. H. Schmidt, M. Schöffler, A. Czasch, W. Schöllkopf, R. E. Grisenti, T. Jahnke, D. Blume, R. Dörner, Science 348, 551 (2015)

12. D. Bressanini, J. Phys. Chem. A 118, 6521 (2014)

13. P. Stipanović, L. Vranješ Markić, J. Boronat, J. Phys. B: At. Mol. Opt. Phys. 49185101 (2016)

14. H. Suno, J. Phys. B: At. Mol. Opt. Phys. 49, 014003 (2016)

15. J. Yuan, C. D. Lin, J. Phys. B, 31, L637 (1998)

16. I. Baccarelli, G. Delgado-Barrio, F. A. Gianturco, T. GonzalezLezana, S. Miret-Artes, P. Villarreal, Phys. Chem. Chem. Phys. 2, 4067 (2000)

17. A. Delfino, T. Frederico, L. Tomio, J. Chem. Phys. 113, 7874 (2000)

18. I. Baccarelli, G. Delgado, F. A. Gianturco, T. Gonzalez-Lezana, S. Miret-Artes, P. Villarreal, Europhys. Lett. 50, 567 (2000)

19. C. DiPaola, F. A. Gianturco, F. Paesani, G. Delgado BarrioT. Gonzales-Lezana, S. Miret-Artes, P. Villarreal, I. Baccarelli, T. Gonzales-Lezana, J. Phys. B 85, 2643 (2002)

20. Y.Li, Q. Gou, T. Shi, Phys. Rev. A 74, 032502 (2006)

21. H. Suno, B. D. Esry, Phys. Rev. A 82, 062521 (2010)

22. Y. Li, W. Zhang, Q. Gou, H. Song, T. Shi, Phys. Rev. A 82, 022515 (2010)

23. H. Suno, E. Hiyama, M. Kamimura, Few Body Systems 54, 1557 (2013)

24. H. Suno, B. D. Esry, Phys.Rev. A 89, 052701 (2014)

25. P. Stipanové, L. Vranješ Markić, D. Zarić, J. Boronat, J. Chem. Phys. 146, 014305 (2017)

26. J. Boronat, J. Casulleras, Phys. Rev. B 49, 8920 (1994)

27. J. Casulleras, J. Boronat, Phys. Rev. B 52, 3654 (1995)

28. H. Suno, B. D. Esry, Phys. Rev. A 78, 062701 (2008)

29. R. A. Aziz, F. R. W. McCourt, C. C. K. Wong, Mol. Phys. 61, 1487 (1987)

30. U. Kleinekathofer, M. Lewerenz, M. Mladenovic, Phys. Rev. Lett. 83, 4717 (1999)

31. O. Kornilov, Science 348, 498 (2015)

32. J. C. Xie, T. Kar, S. K. Mishra, R.-H. Xie, Chem. Phys. Lett. 593, 77 (2014) 